Instituto Internacional de Investigación y Desarrollo Tecnológico Educativo INDTEC, C.A.

DOI: https://doi.org/10.29394/Scientific.issn.2542-2987.2019.4.12.7.141-161

OAI-PMH: http://www.indteca.com/ojs/index.php/Revista Scientific/oai

Artículo Original / Original Article

\title{
Formación del Pensamiento Reflexivo para el Logro de un Aprendizaje Significativo de la Física en Estudiantes Universitarios
}

\author{
Autoras: Maibelyn del Carmen Acosta Vega \\ Universidad Nacional Experimental "Rafael María Baralt", UNERMB \\ maibelyn19@gmail.com \\ Zulia, Venezuela \\ https://orcid.org/0000-0001-8492-657X \\ Marianny del Carmen Acosta Vega \\ Universidad Nacional Experimental "Rafael María Baralt", UNERMB \\ mariannyacosta3@gmail.com \\ Zulia, Venezuela \\ https://orcid.org/0000-0001-9813-8304
}

\section{Resumen}

Para el estudiante universitario, analizar en base a situaciones que se ejecutan día a día, o sobre aquellas que son determinantes a la hora de tomar alguna decisión, cada vez se vuelve tarea más difícil, en vista de que el ser humano por lo general actúa de manera predictiva, imponiendo su convicción innata y de frente a la praxis tradicional del docente, no pone en práctica su capacidad reflexiva, ya que esta requiere de más tiempo de lo pensado. Cuando surgió el problema se estableció como objetivo primordial, proponer un conjunto de lineamientos para la formación del pensamiento reflexivo en el área de la Física I. Se aplicó una metodología de tipo descriptiva, proyectiva, con diseño no experimental transaccional de campo. Las unidades son estudiantes (61) y docentes (2). Se utilizaron como instrumentos dos cuestionarios. Se validaron mediante la consulta a expertos y su confiabilidad fue de 0.88. El contenido de la investigación comprendió los aspectos más relevantes sobre el desarrollo del Pensamiento Reflexivo y su incidencia en el Aprendizaje en el Área de la física; por ello se consideró pertinente definir lineamientos que permitan el aprendizaje reflexivo para lograr un aprendizaje significativo de la física.

Palabras clave: aprendizaje; física; estudiantes universitarios. 


\title{
Formation of Reflective Thinking for the Achievement of a Significant Learning of Physics in University Students
}

\begin{abstract}
For the College student, analyze based on situations that run every day, or about those who are determining when making a decision, every time becomes more difficult, given that human beings generally acts in a predictive manner, imposing its innate and against conviction to the traditional practice of the teacher, not put into practice their reflective ability, since this requires more time than expected. When it emerged the problem was established as a primary objective, propose a set of guidelines for the formation of reflective thinking in the area of the physics I. A descriptive methodology, projective, transactional non-experimental design of field applied. The units are students (61) and teachers (2). Two questionnaires were used as instruments. They were validated through consultation with experts and its reliability was 0.88 . The content of the research included the most relevant aspects on the development of reflective thinking and its impact on learning in the Area of physics; Therefore it was considered relevant to define guidelines that allow for reflective learning to achieve meaningful learning of physics.
\end{abstract}

Keywords: learning; physics; university students. 


\section{Introducción}

Actualmente la educación se mide por el grado de satisfacción que surgen de las necesidades sociales, evidentemente bajo instrumentos y procedimientos que den confiabilidad a dicha medición, y a su vez se logará evidenciar en la manera que cada estudiante dentro y fuera del salón de clases actué, hable y se desenvuelva en un ambiente común donde la toma de decisiones es de vital importancia para los futuros procederes que determinarán el éxito en la vida; evidentemente todo dentro de procesos reflexivos, dado que nada surge de la nada.

Asimismo, los estudiantes de hoy, presentan infinidad de interrogantes y debido a la tecnología abrumante que los rodea, mediante un simple teléfono inteligente tienen la posibilidad de descargar cualquier aplicación o simplemente con una búsqueda en internet acceden fácilmente al mundo mágico del saber y es allí cuando el estudiante y persona en general reconoce que las universidades, colegios o entes educativos, no es la única dimensión que se tiene para adquirir conocimientos y sobre eso, el hecho que el docente no es el dueño del saber puesto que existen otros espacios que permiten acceder a él.

Por otro lado, Barcia y Carvajal (2015a), exponen que:

Hoy en día, las sociedades del mundo se plantean la importante necesidad de enriquecer la formación cultural del hombre, cuya preparación lo sitúe a la altura del desarrollo del mundo contemporáneo; un hombre culto que comprenda los problemas de su contexto, en su origen y desarrollo, que se inserte en la batalla de ideas, con argumentos sólidos para asumir una actitud transformadora, dirigida al alcance de los ideales sociales de nuestra era (pág. 140).

En base a lo anterior es oportuno preguntarse si las instituciones, los docentes y estudiantes se encuentran cumpliendo el rol que deben cumplir y que si además de ello, se preparan día a día con técnicas y herramientas 
necesarias para lograr una educación de calidad. Hacerse la pregunta y a la vez internalizarla es de suma importancia puesto que de ello dependerá el éxito que los estudiantes obtendrán, promoviendo igualmente un producto con capacidad critico reflexiva, capaz de discernir sobre las habilidades y actitudes que debe desarrollar más allá de lo que establezca un currículo.

Es importante señalar que muchos docentes en diversas áreas, promueven esfuerzos para lograr que sus estudiantes lleguen a un nivel crítico reflexivo más profundo; formando entonces a estudiantes independientes, competentes a la hora de discutir sobre una idea, cuestionar sobre un tema de interés en específico o simplemente razonar sobre un conocimiento adquirido. La dificultad surge cuando los entes universitarios son estudiados desde espacios más profundos y se logra evidenciar que los docentes aun miden la capacidad y el conocimiento del estudiante mediante pruebas y test que solo arrojan una calificación y demuestran a simple vista el grado de difusión de los contenidos expuestos en el salón de clases.

Es así como se muestra a la palestra otra interrogante, donde se deja las destrezas tomadas por los estudiantes, la creatividad, la pericia a la hora de resolver un evaluativo; con todo ello, se logra vislumbrar que las universidades hablan de profundizar sobre una teoría de transformación crítica y reflexiva, pero es evidente que la realidad es otra, que la enseñanza sigue siendo tan tradicional como hace muchos años atrás.

Por su parte Domingo (2013), expone que: "precisamente los planteamientos más avanzados en formación universitaria actual se inclinan hacia propuestas unificadoras de teoría y práctica, que se traducen en proyectos formativos por medio de competencias personales del estudiante" (pág. 35); esto unificación que se propone, sin duda podría propiciar espacios de reflexión en los participantes.

Aunado a lo planteado anteriormente; en las universidades o cualquier ente educativo es probable pensar que el estudiante dentro y fuera del aula de 
clases ya posee herramientas para resolver cualquier tipo de problema y que además es responsable y reflexivo sobre sus propios aprendizajes y que no necesita que se le fomente dicho proceso; pero debe quedar claro que para que el estudiante sea capaz de mostrar autonomía no solo se necesita que se fomenten estrategias tradicionales de cognición y meta cognición sino profundizar con interés e ímpetu en las estrategias metodológicas que emplea cada docente porque realmente allí se encuentra el éxito del proceso enseñanza aprendizaje.

De esta misma manera, Páez (2018), en su artículo Estrategias Constructivistas Aplicadas por el docente para el aprendizaje de la Física en el nivel Superior, afirma que:

Existe en el estudiante de nivel universitario, la posibilidad de presencia de algunas deficiencias en cuanto los conocimientos básicos necesarios para iniciarse en un estudio superior, lo cual pudiera ocasionar, de una forma u otra en éste, dificultades para asimilar y procesar nuevas informaciones a niveles más profundos de conocimiento, aunado a ello el hecho que, quizás el profesor no se interese por diagnosticar la situación presente en el ambiente de clase conduciendo el proceso de enseñanza aprendizaje por la vía menos apropiada, hacia el fracaso formativo del estudiante (pág. 41).

De lo hasta ahora planteado, las autoridades universitarias de todas las entidades, deberían reaccionar y comenzar a emplear métodos, técnicas para favorecer el proceso que llevan a cabo tanto los docentes como los estudiantes; ya se debe derrotar el mito que el estudiante conoce lo que está por venir y que además sabe cómo resolverlo o enfrentarlo; se hace imperante que se mejoren las practicas pedagógicas para ir disminuyendo factores que afectan grandemente al estudiante, uno de ellos quizás el más importante, la frustración, provocando a la vez la ausencia en los salones de clases, ya que el estudiante de frente con tan bajo rendimiento considera que no es capaz de sobrellevarlo y prefiere desertar. 
En este sentido, las instituciones educativas deben ser más que una estructura de bloque y concreto donde se congregan una cantidad de persona, unas con el fin de impartir o propiciar el conocimiento y otras que solo estén para recibirlo; ese proceso debe ir más allá, puesto que en los espacios educativos es donde se forman verdaderos transformadores de la sociedad que promulgaran dentro de espacios reflexivos teorías, conocimientos que más adelante irán en pro a la misma sociedad.

Por otro lado, las asignaturas de competencia lógico-matemática están presentes en los programas de estudio, sobre todos en las carreras relacionadas con la ingeniería, donde se les asignan un alto porcentaje de horas académicas, en la que los estudiantes deben poseer o tener desarrollada la destreza de representar y generalizar, además de fomentar la poderosa la agilidad para resolver problemas.

Exactamente dentro de esas asignaturas de competencia, las de orden matemático, la física se vuelve materia obligatoria en las distintas carreras de profesionalización de la ingeniería, por lo que debería hacerse esfuerzos para mejorar su enseñanza, sin embargo, se logra evidenciar como ya se ha mencionado, que las clases dentro de los espacios colaborativos en las instituciones se reproducen de manera tradicional, en la cual el proceso de enseñanza aprendizaje pareciera se da en una sola dirección, y el estudiante es solo capaz de recibir la información sin proyectarse y pensar reflexivamente.

De lo anterior se podría referir que el interés que los profesores dan a la enseñanza de estas materias, no garantiza el éxito en el participante, ya que en ocasiones presentan bajo rendimiento, como lo muestra la estadística de aprobados y reprobados del programa Ingeniería en la catedra Física I, de la Universidad Alonso de Ojeda, data del primer al tercer académico del año 2015; dichos datos fueron obtenidos mediante un estudio previo; lo que conlleva a referir que el profesor o facilitador debe dedicar aún más tiempo a 
su enseñanza; de manera que, el participante actual, pasará a actuar de manera reflexiva ante los problemas que se le presentan.

Por todo lo expuesto anteriormente, se emprendió una investigación basada en el pensamiento crítico reflexivo; haciendo énfasis en aquellos docentes que dictan la catedra Física I y participantes que cursan la catedra en la Universidad Alonso de Ojeda, Sede Ingeniería, donde el pensamiento reflexivo se forma mediante la participación pedagógicas reflexivas, el cual se ponen en evidencia mediante la forma en que se es capaz de expresar y actuar y basada en la experiencia laboral de las autoras como docente, se logra evidenciar luego de un proceso de observación directa y en base a la data proporcionada por la Universidad Alonso de Ojeda, que los estudiantes de esta institución presentan un bajo rendimiento, quizás propiciado por falta de espacios colaborativos-reflexivos.

Asimismo, es notable como los estudiantes prefieren los procesos memorísticos a la hora de analizar situaciones o resolver ciertos problemas, sienten comodidad cuando las respuestas que pueden plasmar en un evaluativo son replica de las vistas en clases o plasmadas en libros y cuadernos; así pues, si son problemas de índole numérico la resolución mediante procedimientos netamente mecánicos es zona de confort y sienten confianza de recibir una buena calificación.

Este planteamiento hay que vislumbrarlo como una problemática dentro de la educación, puesto que los estudiantes deben ser formados de manera activa para que en cualquier ámbito él sea capaz de tomar decisiones y como cada día recibe más información por diversos medios, debe poseer herramientas necesarias para lograr discernir.

Por todo lo anterior expuesto, se busca suprimir todos los problemas que afectan el proceso de enseñanza-aprendizaje en los estudiantes, de modo que existan alternativas y a su vez estrategias que permitan desarrollar el pensamiento reflexivo; sólo de esa manera se podría hablar de una educación 
que realmente aporta beneficios a la sociedad.

\section{Fundamentación teórica}

\subsection{Pensamiento Reflexivo}

Pensar reflexivamente podría representar una necesidad para la sociedad actual y son muchos los aportes investigativos que se han hecho al respecto; se inicia con la postura de Abella et al. (2018), para ellos el proceso de pensamiento reflexivo es todo aquello que:

permite establecer una relación analítica con la acción que se realiza y favorece el empleo deliberado, consciente, sistemático de nuestros recursos mentales en dirección a un propósito. Es un pensamiento instrumental y convierte los procesos espontáneos de pensamiento en una reflexión que guía la cognición hacia la acción (pág. 17).

En el mismo orden de idea, se puede añadir a lo planteado por los autores, que el proceso de pensar reflexivamente pertenece a un proceso cognitivo, cuyas actividades requieren amplio grado de originalidad por parte del pensante, las cuales estarán sustentadas por los diversos principios de la praxis reflexiva. Así mismo también es importante resaltar la postura de da Costa, Bertacchini y Alves (2016), ellos manifiestan que:

la literatura sobre el pensamiento reflexivo tiene raíces en dos disciplinas académicas: la filosofía y la psicología. No obstante, también se observa una tercera vertiente en el campo de la educación. En la filosofía, se busca definir al pensador crítico hipotético a partir de sus cualidades y características, y en la psicología se procura describir al pensador crítico con base en sus habilidades o acciones. En el ámbito de la educación, existe un enfoque en la enseñanza y en la evaluación de las habilidades del pensamiento reflexivo (pág. 2).

Esta postura fue relevante puesto que de ella se manifestaron aspectos tales como que la lógica y el análisis provienen de conceptos filosóficos y matemáticos, mientras que pensar de manera racional y objetiva implica 
procesos más amplios que tienen origen en la psicología y la sociología y que

pensar de manera crítica reflexiva implica hacerlo de manera integral.

Luego de exponer referentes teóricos, se logra dilucidar qué pensar de manera crítica reflexiva es un proceso dinámico el cual requiere de conocimientos previos y confrontados con los conocimientos adquiridos se hará un análisis y se llegará a una postura de racionamiento justificado; postura que en ocasiones rompe paradigmas. Los conocimientos previos son de relevancia, pero no determinantes, la práctica y la metodología guiarán el proceso e indicará cómo usar la inteligencia y el conocimiento previo para alcanzar posturas más racionales con la información ya obtenida, ya que el pensamiento reflexivo debe ser una habilidad que se desarrolla y se demuestra.

\subsection{Aprendizaje Significativo}

El ser pensante puede aprender de muchas maneras, pero cuando lo hace englobando dimensiones como la emocional, motivacional y cognitiva, se dice que el aprendizaje es significativo, al producirse este se crean modelos mentales y el tiempo y las vivencias determinaran la manera en que se ve y se gestiona la información. No se puede confundir el aprendizaje significativo con el aprendizaje repetitivo, este último es memorístico y lo aprendido se recordará por un periodo muy corto de tiempo.

Al respecto, Ausubel señalaba que aprender de manera significativa se le atribuye a la acción por parte del estudiante de incorporar un nuevo conocimiento a su estructura cognitiva, pero no de forma arbitraria, sino por el contrario, de manera ordenada, relacionándolo con el conocimiento previo adquirido; así mismo expresaba que para que este proceso se realice es necesario cumplir con dos condiciones básicas; la primera hace referencia a que el contenido que el participante se dedique a estudiar debe ser de total interés para él y la segunda viene dada por la disposición que tenga el 
estudiante para relacionar sustancialmente el material actual a su estructura cognitiva.

Por otro lado, Chacín (2015), expone que: "sólo habrá aprendizaje significativo cuando lo que se trata de aprender se logra relacionar de forma sustantiva y no arbitraria con lo que ya se conoce quien aprende, es decir, con aspectos relevantes y preexistentes de su estructura cognitiva" (pág. 37). Asimismo, Carranza (2017), manifiesta que: "se puede asegurar que para que el aprendizaje significativo pueda concretarse, se requiere entonces una serie de acciones y/o cogniciones en cada uno de los estudiantes. Dichas acciones pueden denominarse dimensiones del aprendizaje significativo" (pág. 903).

En líneas generales el aprendizaje significativo no es más que un aprendizaje que se da con sentido lógico, racional y justificable y que se trata solo de relacionar el conocimiento previo del estudiante con el conocimiento actual y que para que esto se dé el docente debe crear estrategias que le permitan a los estudiantes entrar en confort para que así esté dispuesto y motivado a aprender.

\subsection{Aprendizaje en la Educación Superior}

Los sistemas de educación superior están siendo estudiados desde diversas perspectivas y cada una de ellas apunta y eleva la presión para que sea aumentada la calidad en la enseñanza, esta situación va desde niveles mundiales hasta casos en específico que se puedan estudiar. Sin duda uno de los rasgos más predominantes en este nivel superior es la búsqueda de aprendizajes significativos, verdaderamente ambiciosos y profundos, puesto que se habla de estudiantes en un nivel donde se asume la educación formal y por ende las universidades deben de encargarse de formar a estudiantes verdaderamente de alto nivel y con capacidad autónoma, sin importar la disciplina o especialidad que se curse. Tal como lo plantean Barcia y Carvajal (2015b): 
En sí, se debe considerar la necesidad de organizar adecuadamente las actividades interactivas de aprendizajes en la formación superior, que se admita las relaciones comunicativas para promover la cultura del diálogo y el debate, donde puedan expresarse libremente, lo que propiciará su crecimiento como personas y permitirá el redimensionamiento del carácter activo, creador y humano del nuevo profesional, donde el trabajo en el grupo es de vital importancia ya que es ahí donde se elaboran los conocimientos (pág. 143).

En este sentido, los docentes le brindan a sus estudiantes los argumentos necesarios según la carrera que curse, es decir en las carreras de ingeniería donde la lógica el análisis y la toma de decisiones es importante, dan dominio factual a los hechos y a lo que determine fielmente una metodología; por otro lado, las carreras humanísticas profundizan en el desarrollo personal y de las habilidades de los estudiantes; es aquí un punto importante dado que si buscan estudiantes verdaderamente reflexivos, se deben consolidar estudiantes integrales.

Para la mayoría de los estudiantes universitarios, su experiencia por la casa de estudio correspondiente viene dada por un paseo en una malla curricular, en la cual va absorbiendo los aprendizajes que le son de su agrado $y$ los que no, los estudia pero de manera pasiva pero sin enfatizar y comprender lo visto, solo lo hace de manera memorística y si ahondamos más, cuando se trata del estudio de materias de orden lógico matemático, el estudiante es muy mecánico, no analiza los problemas si no que busca las respuestas según un patrón dado en clases.

Todo lo planteado representan problemas que hay que anularlos de los sistemas educativos; los docentes requieren de un cambio profundo y deben profundizar en el modo de enseñanza para lograr que los estudiantes comprendan los fenómenos de igual manera que lo hacen los expertos en el tema. En este sentido, será un buen docente aquel que busque profundizar en sus estrategias, diseñe cursos y metodologías, que introduzca los medios de 
comunicación e información de manera adecuada para que el estudiante conozca diversos espacios colaborativos de conocimiento para lograr el verdadero aprendizaje significativo.

Asimismo, debe tomarse en consideración las características de la población estudiantil, estando consciente que son de tipo heterogénea y a veces hasta numerosas; abordar estos aspectos permitirá acceder a estudiantes a tiempo completo, estudiantes con dominio y de calidad, que harán énfasis en la reflexión en cada proceso de enseñanza aprendizaje.

\section{Metodología}

El propósito de la investigación es describir una situación o un evento, para así establecer lineamientos relacionados con la formación del pensamiento reflexivo por medio de estrategias de enseñanzas para el logro de un aprendizaje significativo de la física I en los estudiantes de Ingeniería de la Universidad Alonso de Ojeda.

Por consiguiente, la investigación se desarrolla bajo el paradigma positivista, bajo investigación de tipo descriptivo, con diseño no experimental, de campo, transaccional ya que el objetivo fundamental fue el de indagar y a su vez describir las variables establecidas. La Muestra tomada fue de (61) estudiantes y (2) docentes de la Facultad de Ingeniería de la Universidad Alonso de Ojeda. Igualmente, se elaboraron instrumentos para la recolección de datos, los cuales fueron, una encuesta, una entrevista no estructurada; así como la observación directa.

De igual manera se empleó la Escala de Lickert, en dos categorías; esto se debió a que el cuestionario fue aplicado tanto a los estudiantes como docentes que representaban la muestra y se optó por un diseño más sencillo para los estudiantes con igual número de categorías para los docentes, dado que los resultados serían comparados en el análisis de datos. 


\section{Análisis de los resultados}

En virtud de las características de los instrumentos empleados para la recolección de datos, la confiabilidad fue determinada por un experto en estadística el cual empleo el coeficiente Alfa de Cronbach dado que este hace referencia a la medición de consistencia de la respuesta del sujeto con respecto a los ítems de los instrumentos. Una vez que se aplicó el coeficiente fueron verificados por el programa estadístico IBM SPSS, el cual arrojó como confiabilidad un valor de 0.88 y 0.99 respectivamente; estos resultados indican que los instrumentos tienen una confiabilidad aceptable.

En este sentido, se presenta información estadística detallada mediante tablas 1, 2, 3 y gráficos 1, 2, 3 de las variables del estudio:

Tabla 1: Resolución de problemas con razonamiento y reflexión.

\begin{tabular}{ccc}
\hline ALTERNATIVAS & FRECUENCIA & PORCENTAJE (\%) \\
\hline Siempre & 20 & 30 \\
A veces & 38 & 58 \\
Nunca & 8 & 12 \\
Total & 66 & 100 \\
\hline
\end{tabular}

Fuente: La Autora (2019).

Gráfico 1: Resolución de problemas con razonamiento y reflexión.

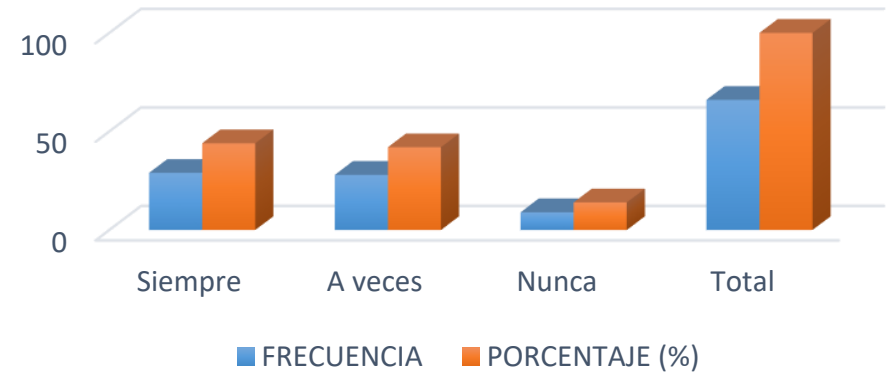

Fuente: La Autora (2019). 
En referencia a las respuestas obtenidas por parte de los estudiantes, el $30 \%$ señala que siempre razona y reflexiona fácilmente al momento de resolver un problema, en sumatoria un $70 \%$ refleja que no propicia espacios de reflexión a la hora de resolver problemas; por lo que se deduce que lo realizan mecánica y reflexivamente, por lo cual no aportaran ideas claras, no logran aprendizajes duraderos y significativos que mejoren su rendimiento académico.

Tabla 2: Empleo de técnicas de aprendizaje por el docente.

\begin{tabular}{ccc}
\hline ALTERNATIVAS & FRECUENCIA & PORCENTAJE (\%) \\
\hline Siempre & 20 & 30 \\
A veces & 36 & 55 \\
Nunca & 10 & 15 \\
Total & 66 & 100 \\
\hline
\end{tabular}

Fuente: La Autora (2019).

Gráfico 2: Empleo de técnicas de aprendizaje por el docente.

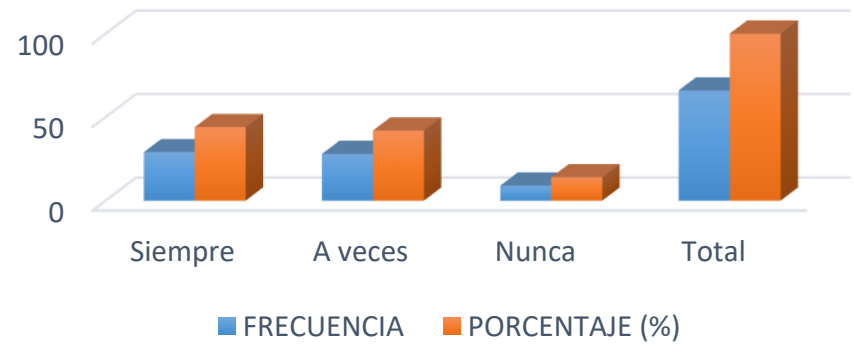

Fuente: La Autora (2019).

En relación a las respuestas obtenidas, 55\% de los estudiantes manifiestan que a veces el docente emplea técnicas de aprendizaje para desarrollar el pensamiento reflexivo, el $15 \%$ dice que nunca y el $30 \%$ responde que siempre. Se puede deducir que los docentes en sus planificaciones consideran técnicas que apoyen el desarrollo del pensamiento reflexivo, pero es probable que lo hagan de manera difusa, dificultando el aprendizaje en el 
área de la física.

Tabla 3: Lineamientos para el pensamiento reflexivo.

\begin{tabular}{ccc}
\hline ALTERNATIVAS & FRECUENCIA & PORCENTAJE (\%) \\
\hline Siempre & 29 & 44 \\
A veces & 28 & 42 \\
Nunca & 9 & 14 \\
Total & 66 & 100 \\
\hline
\end{tabular}

Fuente: La Autora (2019).

Gráfico 3: Lineamientos para el pensamiento reflexivo.

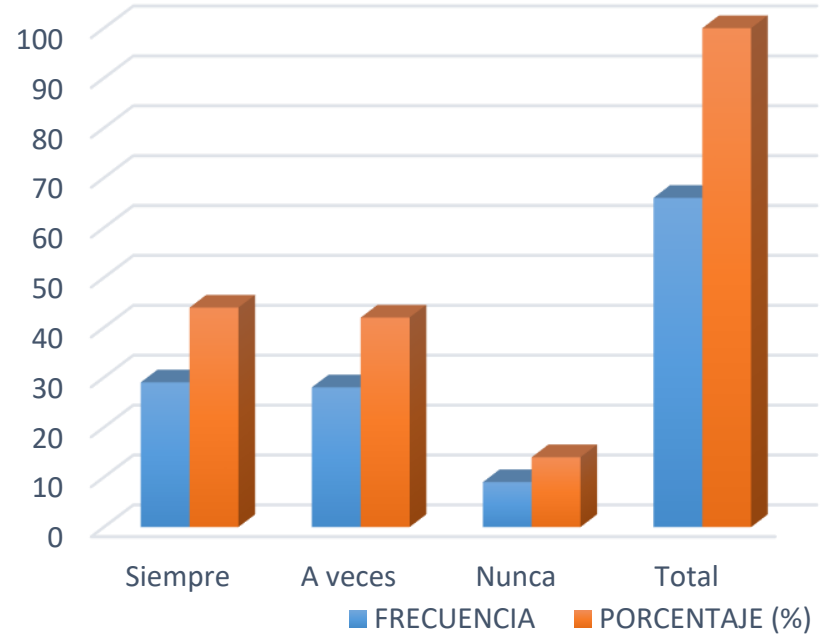

Fuente: La Autora (2019).

Se logra evidenciar el $44 \%$ de los encuestados indican que siempre se debe realizar lineamientos para fortalecer el desarrollo del pensamiento reflexivo en el área de la física, el $42 \%$ señalan que a veces mientras que un 14\% responden que nunca. Cómo se logra evidenciar, la mayoría de los estudiantes responde que son necesarios unos lineamientos para el desarrollo del pensamiento reflexivo en el área de la física para que sus docentes conozcan estrategias y técnicas adecuadas que pueda ser aplicada en el aula y de esta manera contribuir a un aprendizaje significativo. 


\section{Conclusiones}

En base a los objetivos planteados en la investigación y los resultados obtenidos, descritos estadísticamente, es oportuno concluir sobre varios aspectos que involucran las variables en estudio, como son, el pensamiento reflexivo y su incidencia en el aprendizaje significativo de la Física.

En primer lugar, se logra evidenciar que los estudiantes ante la resolución de ejercicios de orden lógico matemático, no reflexionan ante los mismos, todo lo contrario, aplica procedimientos netamente mecánicos. De lo anterior, se deduce que son muchos los obstáculos por debatir cuando se pretende incluir en el ámbito del pensamiento crítico reflexivo el ejercicio de una verdadera enseñanza de la Física.

Uno de ellos y el que más discusiones ha traído a la palestra, es el hecho que el estudiante no le encuentra sentido a la carrera que estudia, en primera instancia porque lo hace de manera ideológica y ya cuando se desarrollan los contenidos no le encuentra relación con ningún evento que él vaya a vivir, es decir para ellos el conjunto de teorías y fórmulas que se desarrollan en los espacios colaborativos son solo para los científicos, que en el campo laboral nada de ese contenido será relevante para desarrollar su trabajo.

En segundo lugar, se logra evidenciar que los docentes aplican escazas estrategias, técnicas y /o herramientas que permitan propiciar espacios de reflexión en los salones de clases. De lo descrito se dilucida que ser docente, contar con un cargo en cualquier ente institucional y pensar que se tienen los conocimientos básicos para impartir la cátedra de Física a cierto grupo de estudiantes, no representan los requisitos básicos para generar conocimientos que validen un verdadero proceso de enseñanza aprendizaje.

Sin duda, se debe ir más allá del saber pedagógico del docente y tener en cuenta que es algo que transciende los muros de una universidad, que realmente se refuerza en la práctica y que el docente tiene la responsabilidad 
y la necesidad de contribuir en la formación de seres humanos que difícilmente se conjuguen todos bajo una comunidad científica.

En tercer lugar, es importante señalar que se hace imperante la necesidad de proponer una serie de lineamientos que permitan fomentar el pensamiento reflexivo para el logro del aprendizaje significativo de la fisca en estudiantes de la Universidad Alonso de Ojeda. En virtud a lo planteado es importante iniciar el proceso desde el inicio mismo de la carrera, donde cada tema estudiado logre responder a algún tipo de inquietud o genere expectativa en el estudiante.

Así mismo, los espacios para pensar reflexivamente deben ser fomentados, así se logra que aumente la curiosidad y el interés por cuestionar cada situación que exista o coexista en un ambiente determinado, recurriendo a la vez a aumentar el nivel de creatividad e innovación en pro del potencial critico reflexivo.

No obstante, es importante señalar que en los espacios donde se fomenta el pensamiento reflexivo, la exposición oral se hace sentir, pero es un error pensar que en ese proceso es el docente el cual debe hablar sin fin y el estudiante es solo un receptor que en su silencio es activo porque debería estar reflexionando sobre lo escuchado. Los contenidos de las sesiones en cada encuentro deben mostrar actividad por parte del docente y el estudiante y generar reflexión que sea manifestada de manera individualizada donde cada uno tome la situación, la indague y analice como objeto de su propio pensamiento.

El racionamiento inductivo lleva manifestar finalmente que existen algunas estrategias que logran fomentar la práctica reflexiva en el ámbito universitario; tales como el dialogo reflexiva, la interrogación didáctica y empleando la pregunta retórica. Todas ellas puestas en práctica elevan el nivel reflexivo en los participantes, logrando analizar los problemas planteados en relación a la física, para así reflexionar sobre ellos y resolver no de manera 
mecánico como es la costumbre.

\section{Recomendaciones}

- Incentivar a los estudiantes cursantes de la cátedra Física, que fortalezca el pensamiento reflexivo tanto en el salón de clases como fuera de él, para que los aprendizajes estén interrelacionados con el entorno de la vida diaria.

- Motivar al personal docente a la investigación, selección y manejo de planteamientos que sean del agrado e incentiven la curiosidad por las materias de orden lógico matemático para lograr aprendizajes duraderos, significativos y comprensivos; es decir que cree una disposición favorable del estudiante para aprender.

- Poner en práctica los lineamientos para fomentar el pensamiento reflexivo, de modo que el docente pueda crear espacios en donde los estudiantes, puedan adquirir hábitos de reflexión y creatividad, como por ejemplo, rincones de trabajo de lectura, problemas, juegos de razonamiento, olimpiadas de orden matemático, de esta manera desarrollar el pensamiento reflexivo de los estudiantes.

\section{Referencias}

Abella, V., Alós, M., Anglés, R., Ausín, V., Belén, A., Cejas, R... \& Vidal, J. (2018). El Pensamiento reflexivo a través de las Metodologías Narrativas: Experiencias de Innovación en Educación Superior. Cuaderno de Docencia Universitaria 35, ISBN: 978-84-17667-02-3. Barcelona, España. Ediciones Octaedro. Recuperado de: http://diposit.ub.edu/dspace/bitstream/2445/126271/1/CDU-35.pdf Barcia, J., \& Carvajal, B. (2015). El Proceso de Enseñanza Aprendizaje en la Educación Superior. Revista Electrónica Formación y Calidad Educativa REFCalE, 3(3), 139-154, ISSN: 1390-9010. Recuperado de: 
http://runachayecuador.com/refcale/index.php/refcale/article/view/57/6

$\underline{22}$

Carranza, M. (2017). Enseñanza y Aprendizaje significativo en una modalidad mixta: Percepciones del docente y estudiantes. Ride Revista Iberoamericana para la Investigación y el Desarrollo Educativo, 8(15), 898-922, ISSN: 2007-7467. Recuperado de:

https://dx.doi.org/10.23913/ride.v8i15.326

da Costa, F., Bertacchini, L., \& Alves, V. (2016). Pensamiento crítico: análisis del concepto bajo la óptica evolucionista de Rodgers. Revista Latino-Americana de Enfermagem RLAE, 24, 1-12, On-line version ISSN: 1518-8345. Recuperado de:

http://dx.doi.org/10.1590/1518-8345.1191.2785

Chacín, F. (2015). Estrategias Didácticas para el aprendizaje significativo de la asignatura Clínica del Niño y del Adolescente. Trabajo Especial de Grado. Valencia, Venezuela: Universidad de Carabobo. [Documento en línea]. Recuperado de:

http://mriuc.bc.uc.edu.ve/bitstream/handle/123456789/2156/fchacin.pdf ?sequence $=1$

Domingo, A. (2013). Practica Reflexiva para los docentes. De la reflexión ocasional a la reflexión sistemática. Revista de Docencia Universitaria, 11(3), 1-420, ISSN: 1887-4592; ISBN: 978-3-639-55345-

1. Recuperado de:

https://polipapers.upv.es/index.php/REDU/article/view/5542/5533

Páez, A. (2018). Estrategias Constructivistas Aplicadas por el Docente para el Aprendizaje de la Física en el Nivel Superior. Revista Scientific, 3(7), 37-56. Recuperado de:

https://doi.org/10.29394/Scientific.issn.2542-2987.2018.3.7.2.37-56 


\section{Maibelyn del Carmen Acosta Vega}

e-mail: maibelyn19@gmail.com

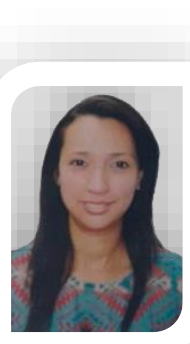

Nacida en Cabimas, Estado Zulia, Venezuela, el día 21 de octubre del año 1984. Ingeniera en Mantenimiento Mecánico; Magister Scientiarum en Docencia para la Educación Superior, con Componente Docente. Con experiencia docente desde hace más de 11 años. Actualmente Docente a tiempo completo en la Universidad Nacional Experimental "Rafael María Baralt" (UNERMB). 


\section{Marianny del Carmen Acosta Vega}

e-mail: mariannyacosta3@gmail.com

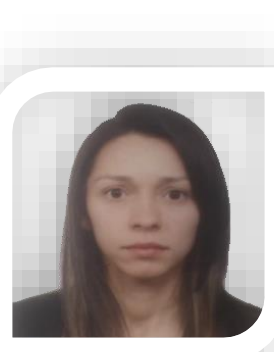

Nacida en Cabimas, Estado Zulia, Venezuela, el día 29 de noviembre del año 1986. Ingeniera en Mantenimiento Mecánico; Magister Scientiarum en Docencia para la Educación Superior, con Componente Docente. Con experiencia docente desde hace más de 9 años. Actualmente Docente a tiempo completo en la Universidad Nacional Experimental "Rafael María Baralt" (UNERMB).

El contenido de este manuscrito se difunde bajo una Licencia de Creative Commons ReconocimientoNoComercial-Compartirlgual 4.0 Internacional 